\title{
Review Article \\ The Outline of Prognosis and New Advances in Diagnosis of Oral Squamous Cell Carcinoma (OSCC): Review of the Literature
}

\author{
Esam Ahmad Omar \\ Taibah University, P.O. Box 40361, Almadinah 41499, Saudi Arabia \\ Correspondence should be addressed to Esam Ahmad Omar; esamomar@hotmail.com
}

Received 7 May 2013; Accepted 12 July 2013

Academic Editor: Shun-Fa Yang

Copyright (C) 2013 Esam Ahmad Omar. This is an open access article distributed under the Creative Commons Attribution License, which permits unrestricted use, distribution, and reproduction in any medium, provided the original work is properly cited.

\begin{abstract}
Objective. Oral squamous cell carcinoma (OSCC) has a remarkable incidence over the world and a fairly strenuous prognosis, encouraging further research on the prognostic factors and new techniques for diagnosis that might modify disease outcome. Data Sources. A web-based search for all types of articles published was initiated using Medline/Pub Med, with the key words such as oral cancer, prognostic factors of oral cancer, diagnostic method of oral cancer, and imaging techniques for diagnosis of oral cancer. The search was restricted to articles published in English, with no publication date restriction (last update April, 2013). Review Methods. In this paper, I approach the factors of prognosis of OSCC and the new advances in diagnostic technologies as well. I also reviewed available studies of the tissue fluorescence spectroscopy and other noninvasive diagnostic aids for OSCC. Results. The outcome is greatly influenced by the stage of the disease (especially TNM). Prognosis also depends or varies with tumour primary site, nodal involvement, tumour thickness, and the status of the surgical margins. Conclusion. Tumour diameter is not the most accurate when compared to tumour thickness or depth of invasion, which can be related directly to prognosis. There is a wide agreement on using ultrasound guided fine needle aspiration biopsies in the evaluation of lymph node metastasis.
\end{abstract}

\section{Introduction}

Head and neck malignancies constitute approximately 5\% of all malignant tumors of the body [1]. Squamous cell carcinoma represents almost $95 \%$ of the head and neck cancers. The incidence of oral cancer varies from region to another in the world. The most common oral sites involved vary as well from region to another. It appears that the geographic location has no role with this rather than it is the oral habits popular in certain countries which vary from region to another. In certain countries, such as Sri Lanka, India, Pakistan, and Bangladesh, oral cancer is the most common type of malignancy. In India oral cancer represent more than $50 \%$ of all malignancy reported. The high incidence rate in these countries may relate to specific oral habits such as Betel and similar habits. Approximatively $30-35 \%$ of the tumors (OSCC) occur a tongue, $20-25 \%$ at the gum, $5-7 \%$ at the floor of the mouth, $4-6 \%$ at the soft palate and only $2-3 \%$ at the cheeks [2]. The tumors were usually diagnosed at the ages between 50 and 79 years, $96,6 \%$ being over 40 years old. Generally, Oral cancer incidence rates have overall increased since the mid-1970s, with most of this increase occurring since the late 1980s for both sexes. European incidence rates have increased by $25 \%$ and $28 \%$ for men and women, respectively, in the last decade particularly in years between 1998-2000 and 2007-2009. The mortality of oral carcinoma varies widely between African-Americans and Caucasians and the ethnicity type may play a role in the survival rates. The overall survival of patients has not improved significantly during the past 20 years, with 5 -year survival rates between $45-50 \%$.

\section{Etiology}

(i) Cigarette smoking: compared with persons who do not smoke, the risk of oral cancer in persons who smoke low/medium-tar cigarettes and high-tar cigarettes was 8.5- and 16.4-fold greater, respectively [3].

(ii) Alcoholic beverages may contain carcinogens or procarcinogens, including nitrosamine and urethane contaminants and ethanol. Ethanol is metabolized 
by alcohol dehydrogenase and, to some extent, by cytochrome P450 to acetaldehyde, which may be carcinogenic [3].

(iii) Betel and similar habits [4]: the betel quid contains a variety of ingredients, including betel vine leaf, betel (areca) nut, catechu, and, often, slaked lime together with tobacco. Some persons chew the nut only, and others prefer paan, which includes tobacco and sometimes lime and catechu. In 1986, the International Agency for Research on Cancer has deemed betelquid chewing an important risk factor, and the areca (betel) nut habit with or without tobacco use can cause cytogenetic changes in oral epithelium. Various other chewing habits, usually combinations that contain tobacco, are used in different cultures (e.g., Qat, Shammah, and Toombak). Tobacco chewing by people from parts of Asia appears to predispose to OSCC, particularly when it is started early in life and is used frequently and for prolonged periods $[3,5,6]$. Studies from India have confirmed the association between paan tobacco chewing and OSCC, particularly cancer of the buccal and labial mucosa [3].

(iv) Diet: a significant protective effect of diet against oral cancer has generally been shown in persons who consume beta-carotene-rich vegetables and citric fruits [3].

(v) Mouthwash use: the effect of the alcohol in mouthwash appears to be similar to that of alcohol used for drinking although the contribution of mouthwash use to oral cancer must be small in terms of attributable risk. This controversy continues $[7,8]$.

(vi) Socioeconomic status: behaviors that lead to social instability or social instability itself have been linked to an increased risk of oral cancer, but many other explanations may exist (e.g., habits, oral health, diet, and nutrition) [7].

(vii) Infective agents: Candida albicans and viruses, such as herpes viruses and papillomaviruses, may be implicated in some cases. Human papillomaviruses are particularly implicated in oropharyngeal cancers $[3$, 9].

(viii) Others: associations also are apparent between oral cancer and other various oral conditions (e.g., oral submucous fibrosis, oral lichen planus, lupus erythematosus, dyskeratosis congenita, and Fanconi anemia) [3].

Smoking and alcoholism are dominant risk factors, being found in the past history of $75 \%$ of the patients with oral cavity cancers. A frequency of 2-4 times greater of squamous carcinoma in smokers has been observed, and the risk is rising up to 6-15 times in association with chronic alcoholism $[10,11]$. Epidemiological studies showed a high risk for this disease for the families with a history of head and neck cancers [12].

\section{Precancerous Lesions}

(i) Leukoplakia.

(ii) Erythroplakia.

(iii) Oral submucous fibrosis.

(iv) Oral lichen planus.

(v) Chronic hyperplastic candidiasis (candida leukoplakia).

(vi) Syphilis.

(vii) Actinic keratosis.

(viii) Sideropenic dysphagia.

(ix) Palatal changes associated with reverse smoking (leukokeratosis nicotina palatinae).

(x) Discoid lupus erythematosus.

(xi) And others.

Prognostic Factors

(a) Patient's factors:

(1) Age.

(2) Sex.

(3) General condition of the patient and preoperative haemoglobin level.

(4) Tolerance.

(5) Occupation.

(6) Acceptance and compliance.

(7) Lifestyle.

(8) Socioeconomic status.

(b) Tumour's factors:

(1) Size.

(2) Site.

(3) Locoregional metastasis.

(4) Histology of tumour.

(5) Degree of differentiation.

(6) Margin of the tumour after surgery.

(7) Thickness of the tumour (degree of invasion).

(8) Volume of the tumour.

(9) Speed of the growth which may reflect its biological behavior.

(c) Team's factors:

(1) It is affected by the patient's factors and tumour's factors.

(2) The ability of the center and its facilities.

(3) Skills of the surgeons and their experience.

(4) Skills of radiotherapists and chemotherapists and their experience.

(5) Availability and skills of dental and prosthetic services.

(6) Availability and skills of rehabilitation services. 
TABLE 1: T-primary tumour.

\begin{tabular}{ll}
\hline TNM & FIGO \\
TX & Primary tumour cannot be assessed \\
T0 & No evidence of primary tumour \\
Tis & Carcinoma in situ \\
T1 & Tumour $2 \mathrm{~cm}$ or less in greatest dimension \\
T2 & Tumour more than $2 \mathrm{~cm}$ but not more than $4 \mathrm{~cm}$ in greatest dimension \\
T3 & Tumour more than $4 \mathrm{~cm}$ in greatest dimension \\
T4a (lip) & Tumour invades through cortical bone, inferior alveolar nerve, floor of mouth, or skin (chin or nose) \\
T4a (oral cavity) & Tumour invades through cortical bone, into deep/extrinsic muscle of tongue (genioglossus, hyoglossus, \\
T4b (lip and oral cavity) & palatoglossus, and styloglossus), maxillary sinus, or skin of face \\
\hline
\end{tabular}

Note: superficial erosion alone of bone/tooth socket by gingival primary is not sufficient to classify a tumour as T4.

(7) Availability and skills of supportive services (skills and training of nursing staff, speech therapist, clinical psychologist, and oral hygienist).

The outcome is greatly influenced by the stage of the disease, the TNM classification, the tumour's size, and locoregional metastasis (TNM) (Tables 1, 2, 3, and 4) [13].

\section{Methods}

A web-based search for all types of articles published was initiated using Medline/PubMed, with the key words such as oral cancer, prognostic factors of oral cancer, diagnostic method of oral cancer, and imaging techniques for diagnosis of oral cancer. The search was subsequently refined. The sites of specialized scientific journals in the areas of oral and maxillofacial surgery, oral medicine, and oncology were also used. I give an overview of published studies on the prognostic factors of OSCC and diagnostic aids, emphasizing on noninvasive diagnostic aids and the functional diagnostic imaging that can be used in diagnosis of OSCC. The search was restricted to articles published in English, with no publication date restriction (last update April, 2013).

\section{Review of the Literature}

3.1. Site. The most commonly reported oral cancer sites include the floor of the mouth (FOM) and lateral borders of the tongue. The tongue, as a whole, is the most common (40-50\%) site for oral SCC in European and American population. Asian population usually suffers from cancer of the buccal mucosa due to betel quid/tobacco chewing habits; buccal mucosa SCC constitutes 40\% of OSCC in Sri Lankan population [14].

There is a wide belief that involvement of anterior site is usually associated with better prognosis than the posterior site. Five-year survival is significantly reduced for more posteriorly located tumours (i.e., oropharyngeal compared to oral) [15]. Reduction in survival is largely explained by tumour's site influence on nodal metastasis [16]; this can be linked to the lymphatic drainage of these locations and ability of locoregional surgical management. The lymphatic
TABLE 2: N-regional lymph nodes.

\begin{tabular}{|c|c|}
\hline $\mathrm{NX}$ & Regional lymph nodes cannot be assessed \\
\hline N0 & No regional lymph node metastasis \\
\hline N1 & $\begin{array}{l}\text { Metastasis in a single ipsilateral lymph node, } 3 \mathrm{~cm} \text { or less } \\
\text { in greatest dimension }\end{array}$ \\
\hline $\mathrm{N} 2$ & Metastasis as specified in $\mathrm{N} 2 \mathrm{a}, 2 \mathrm{~b}, 2 \mathrm{c}$ below \\
\hline $\mathrm{N} 2 \mathrm{a}$ & $\begin{array}{l}\text { Metastasis in a single ipsilateral lymph node, more than } \\
3 \mathrm{~cm} \text { but not more than } 6 \mathrm{~cm} \text { in greatest dimension }\end{array}$ \\
\hline $\mathrm{N} 2 \mathrm{~b}$ & $\begin{array}{l}\text { Metastasis in multiple ipsilateral lymph nodes, none } \\
\text { more than } 6 \mathrm{~cm} \text { in greatest dimension }\end{array}$ \\
\hline $\mathrm{N} 2 \mathrm{c}$ & $\begin{array}{l}\text { Metastasis in bilateral or contralateral lymph nodes, } \\
\text { none more than } 6 \mathrm{~cm} \text { in greatest dimension }\end{array}$ \\
\hline N3 & $\begin{array}{l}\text { Metastasis in a lymph node more than } 6 \mathrm{~cm} \text { in greatest } \\
\text { dimension }\end{array}$ \\
\hline
\end{tabular}

Note: midline nodes are considered ipsilateral nodes.

TABLE 3: M-distant metastasis.

\begin{tabular}{ll}
\hline MX & Distant metastasis cannot be assessed \\
M0 & No distant metastasis \\
M1 & Distant metastasis \\
\hline
\end{tabular}

TABLE 4: Stage grouping.

\begin{tabular}{lccc}
\hline Stage 0 & Tis & N0 & M0 \\
Stage I & T1 & N0 & M0 \\
Stage II & T2 & N0 & M0 \\
Stage III & T1, T2 & N1 & M0 \\
& T3 & N2, N1 & M0 \\
Stage IVA & T1, T2, T3 & N0, N1, N2 & M0 \\
& T4a & N3 & M0 \\
Stage IVB & Any T & Any N & M0 \\
Stage IVC & T4b & Any N & M1 \\
\hline
\end{tabular}

drainage of posterior part of the tongue is bilateral while anterior part of the tongue is unilateral, and in general the anterior part of the oral cavity drains at the upper part of cervical lymph node while the posterior part drains more 
inferiorly, and it is well known that involvement of the upper part of lymph node is usually associated with better prognosis than the inferior part.

3.2. Size. The overall 5-year survival has been reported to vary in range according to tumour size (T1/T2 commonly referred to as "low-risk tumours" and T3/T4 commonly referred to as "high-risk"). The outcome is greatly influenced by the stage of the disease (especially TNM) [17]. Prognosis also depends or varies with tumour primary site, nodal involvement, tumour thickness, and the status of the surgical margins. Moreover, the cumulative effects of tobacco, betel nut, and alcohol decrease the survival rate [18]. Soo in 1988 reported on 283 of squamous cell carcinoma; he found $30 \%$ diagnosed at $\mathrm{T} 1,48 \%$ at $\mathrm{T} 2,17 \%$ at $\mathrm{T} 3$, and $11 \%$ at $\mathrm{T} 4$. $\mathrm{He}$ reported $95 \%$ of the cases required resection, $32 \%$ required marginal resection, and $63 \%$ required segmental resection. The survival rate is closely related to stage of tumour at time of diagnosis. Five-year survival rate is $77 \%$ at stage I, $70 \%$ at stage II, $42 \%$ at stage III, and $24 \%$ at stage IV. The tumour size usually affects choice and outcome of treatment [15]. It also affects the surgeon's ability to achieve complete resection, especially in deep invading tumours. Increased tumour size has been linked to cervical involvement $[16,19]$, high recurrence rate $[16,20]$, and poor prognosis $[21,22]$.

3.3. Tumour Volume. Tumour volume (tumour thickness) is closely related to lymph node metastasis, and it is believed that it reflects the aggressiveness of tumour growth [23]. It is believed that the amount and nature of lymph node metastasis are closely related to size and thickness of tumour. The relationship between thickness of the primary tumour and occurrence of contralateral cervical metastasis was reported to increase by $5 \%$ in T1/T2 SCC of the oral tongue [24], and it is believed that contralateral lymph node involvements have a poorer prognosis than ipsilateral involvement. It has been found that the size of lymph node involvement is closely related to tumour volume (thickness). It is now widely accepted that thickness is more accurate predictor of subclinical nodal metastasis, local recurrence, and survival than tumour size [16]. Tumour diameter is not the most accurate when compared to tumour thickness or depth of invasion, which can be related directly to prognosis. Tongue OSCC of $5 \mathrm{~mm}$ thickness increases the cervical metastasis significantly [25]. Tumour at the floor of the mouth with thickness of $2 \mathrm{~mm}$ should lead to the consideration of elective nodal surgical treatment of the neck [26]. A poor prognosis should be considered if the tumour thickness of buccal mucosa OSCC is $6 \mathrm{~mm}$ or more [19].

3.4. Lymph Node Involvement. Bad prognosis is expected in patients with ipsilateral, contralateral or bilateral nodal involvement. The worst is bilateral then contralateral, then ipsilateral. The size of nodal involvement is a very important determent. The incidence of occult lymph node metastasis in early stage tumours (T1/T2) has been reported to be between $27 \%$ and $40 \%[27,28]$. Extracapsular invasion was identified as an important predictor of regional recurrence, distant metastasis, and, thus, overall survival.

Factors that seem to influence tumour spread to the lymphatics include

(i) tumour primary site (the posterior site is worse than the anterior site of oral cavity);

(ii) tumour thickness (as tumour thickness increased the prognosis became poor);

(iii) double DNA aneuploidy (aneuploidy is an abnormal number of chromosomes);

(iv) poor differentiation (poor differentiation means aggressive biological behaviour).

(a) The pathological aggressiveness of disease (including rapidity of growth, locoregional, and distance spread) is strongly depending on the biological behaviour of the tumour which is not included in TNM classification.

(b) Brandwein in 2005 reported that a weak or limited lymphocyte response at the tumour-host interface is strongly associated with local recurrence and death. Hiratsuka in 1997 reported an adverse relationship between lymphocytic infiltrate and nodal disease and overall prognosis.

Distant metastasis was reported in $5-25 \%$ of OSCC patients [29] most commonly in poor locoregional control. Distance metastasis is usually reported in N2/N3. Extracapsular spread is a very important determent as it significantly increases the risk of distance metastasis.

There is a wide belief that ultrasound guided fine needle aspiration biopsy is best way for evaluation of lymph node involvement. In some studies a $100 \%$ sensitivity in oral SCC has been reported for this method.

Cervical metastasis by a tumour is a firm statement of its aggressive malignant nature. Nothing is more controversial than the management of cervical metastatic disease. It is well recognized that the presence of cervical metastasis is the most important prognostic factor in head and neck squamous cell carcinoma, accounting for a 50\% reduction in the five-year survival rate for ipsilateral cervical lymph node metastasis [30-32] and 75\% reduction in case of bilateral metastasis $[33,34]$. When the risk of metastasis exceeds 15$20 \%$, neck dissection or radiation therapy is indicated $[35,36]$. Most tumours have a predictable pattern of neck metastasis. The main routes of the cervical lymph node metastasis are through the first station nodes [37, 38] Levels I and II, followed by the second station nodes which include Levels III, IV, and V. The metastatic node was seen in Level I (70\%) followed by Level II (66.7\%) and Level III (33.3\%) $[37,38]$. The incidence of false-negative (occult) nodes based on palpation varies in the literature from 27 to $38 \%$. The sensitivity and specificity of palpation are in the range of 60$70 \%[34,35,39]$. Ultrasound guided fine needle aspiration biopsy is a very promising technique in detection of lymph node metastasis. The reported sensitivity of sonography for detecting lymph node metastases ranges from $63 \%$ to $97 \%$ 
and specificity ranges from $74 \%$ to $100 \%$ [40]. The use of FDG-PET scanning in diagnosis of lymph node involvement is controversial and many institutions use MRI or ultrasound guided biopsies rather than PET scans.

3.5. Degree of Differentiation. According to the degree of keratinisation, cellular and nuclear pleomorphism, and mitotic activity, the squamous cell carcinoma is categorised into three categories:

(i) well differentiated,

(ii) moderately differentiated,

(iii) poorly differentiated.

The influence of histologic grading as a prognostic factor in OSCC was assessed in 215 patients by Kademani in 2006, and he found that the degree of differentiation is a significant predictor of locoregional failure and tumour recurrence.

3.6. Invasive Front. Invasive front is a term used for describing the nature of invasion of the tumour at the host-tumour interface as this relation reflects the biological behavior of the tumour. The pattern of invasion can be assessed by using (Anneroth and Bryne) criteria: Grade 1 tumours had welldelineated "pushingor cohesive" borders. In Grade 2, the advancing edge of tumour infiltrated in solid cords, bands, or strands. Grade 3 tumours had margins that contained small groups or cords of infiltrating cells. In Grade 4, there is marked dissociation in small groups or even single cells (noncohesive). Endophytic growth pattern is associated with increased local recurrence. High grades of infiltration (Grade 3 or 4) are usually associated with nodal involvement and subsequent disease metastasis.

3.7. Perineural and Endoneural Invasion. Perineural and endoneural invasion is strongly associated with

(i) tumour size,

(ii) histological grading,

(iii) invasive front,

(iv) nodal involvement.

The perineural spread is affecting overall prognosis and survival [15], because the perineural and endoneural invasion means spreading of the tumour cells up to base of the skull as the tumour cells spread easily though the nerve and surrounding tissues and compartment.

Prognostic value of perineural invasion has been highlighted in several studies and linked to regional recurrence and distant metastasis.

The five-year survival was 53\% during the time period from 1975 to 77 and has increased to $63 \%$ between 1999 and 2005 this difference was found to be statistically significant; using newer diagnostic modalities that detect the disease in its early stage and/or using newer chemotherapeutic options may explain this improvement. Early detection of oral cancer is one of the most efficient ways to reduce the high mortality from this disease. Early detection can minimize the morbidity of the disease and its treatment, which is associated with a severe loss of function, disfigurement, depression, and poor quality of life.

\section{Noninvasive Diagnostic Aids}

(i) Toluidine blue.

(ii) Oral brush biopsy.

(iii) Saliva-based oral cancer diagnosis.

(iv) Light-based detection systems: optical biopsy.

(a) Chemiluminescence (ViziLite Plus; Microlux/ DL, Orascoptic-DK).

(b) Tissue fluorescence imaging (VELscope).

(c) Tissue fluorescence spectroscopy.

(v) Biomarkers:

(a) DNA-analysis.

(vi) Laser capture microdissection.

Invasive Diagnostic Aid

(i) Surgical Biopsy.

3.8. Toluidine Blue (TB) Staining. Toluidine blue (TB) staining is a simple, inexpensive, and sensitive tool for identifying early OSCC and high-grade dysplasias [41]. A 1\% aqueous TB solution is applied for 30 seconds; this acidophilic metachromatic nuclear stain helps to differentiate areas of carcinoma in situ or invasive carcinoma from normal tissue. Toluidine blue is highly sensitive and moderately specific for malignant lesions. The sensitivity is decreased for premalignant lesions with up to $58 \%$ false negative reported for identifying mildto-moderate dysplasia $[42,43]$. Rosenberg and Cretin stated that the sensitivity of toluidine blue staining in oral cancer screening has been found to range from $93.5 \%$ to $97.8 \%$, and to specificity ranged from $73.3 \%$ to $92.9 \%$ [44].

3.9. Oral Brush Biopsy. The accuracy of the brush test has been the subject of many published studies. In every study in which an oral lesion was simultaneously tested with both a brush biopsy and surgical biopsy, this test has been shown to have a sensitivity and specificity well over $90 \%[45,46]$. the brush biopsy collects cells from the full thickness of the oral epithelium. The brush biopsy has so many advantages: it is a chair side, easy to perform, and painless test that can be used to evaluate any suspicious lesion including common small white and red oral lesions to rule out dysplasia. To help localize the optimal site for brushing an abnormality, Gupta in 2004 combined conventional oral brush biopsy with the application of toluidine blue to localize suspect mucosal areas [42]. Scully stated that the sensitivity of brush biopsy in detection of dysplasia or OSCC was $71.4 \%$ but the specificity was only $32 \%$ [47]. 
3.10. Saliva-Based Oral Cancer Diagnosis. Saliva-based oral cancer diagnosis is a noninvasive alternative to serum testing; it is an effective modality for diagnosis, determining prognosis of oral cancer and for monitoring posttherapy status. A significant increase in salivary concentrations of Cyfra 21-1, Tissue Polypeptide-specific Antigen (TPS), and cancer antigen 125 (CA125) was shown with sensitivity of $71 \%$, specificity of $75 \%$, and negative value of $71 \%$ and positive predictive values of $75 \%$. On the other hand Carcinoembryonic antigen (CEA), and cancer antigen 19-9 (CA19-9), did not reach statistical significance $[48,49]$.

3.11. Light-Based Systems. Light-based systems that are based upon the assumption that abnormal metabolic or structural changes have different absorbance and reflectance properties for lights. ViziLite Plus with TBlue system (Zila Pharmaceuticals, Phoenix, Arizona, U.S.), VELscope (LED Dental, White Rock, British Columbia, Canada), Microlux/DL (AdDent Inc, Danbury, Connecticut), and Orascoptic DK (Orascoptic, Middleton, WI) are light-based oral cancer screening aids that have been developed and aimed at assisting in the identification of precancerous and cancerous lesions at their earliest stage. VELscope is a handheld device with an illuminated chemiluminescent light stick that uses visible light in the $430 \mathrm{~nm}$ wavelength in order to cause fluorescent excitation of certain compounds in the tissues. The patients first rinse with acetic acid, and then the oral cavity is examined. In Microlux and Vizilite, the oral cavity is examined with a battery-powered fiber-optic visible light source instead of a chemiluminescent and that requires that the patient first rinses with acetic acid. These devices are not sensitive or specific for diagnosis of any type of abnormal oral lesion. Only a definitive test, examining tissues, can determine the biologic behavior of a lesion [50].

\subsection{Tissue Fluorescence Spectroscopy}

3.12.1. Autofluorescence Spectroscopy. In case of malignancy there are changes in the physical and chemical characteristics of the tissues due to the subcellular architectural changes in cancer, such as nuclear grade and nuclear to cytoplasm ratio, mitochondrial size and density, amount of keratin, and elastin to collagen ratio, and it is well known that all tissues fluoresce and malignant tissues fluoresce less than normal tissues, they have different spectral characteristics. Studies of these methods in normal oral mucosa have shown increased green fluorescence in comparison to neoplastic lesions upon ultraviolet (UV) or near UV light source. Autofluorescence imaging has recently been shown to improve the detection of premalignant and malignant oral lesions. This method is based on the illumination in the absorption of tissue fluorophore molecules $\mathrm{NADH}$ and FAD in the epithelial layer and collagen and elastin in the stroma) in ultraviolet visible spectrum leading to the emission of lower energy photon that can be detected as fluorescence from the oral surface mucosa. Optical fibres may be introduced into the tissues through a hollow needle; the tissue signals are interpreted by spectrometers $[51,52]$. The reported sensitivity in fluorescence spectroscopy technologies was up to $81 \%$ and specificity was $100 \%$ [53].

3.12.2. Enhanced Dye Fluorescence. Photodynamic drug can be applied which enhanced porphyrins such as protoporphyrin IX (protoporphyrin IX is an important precursor to biologically essential prosthetic groups such as heme, cytochrome c, and chlorophylls). The fluorescence is slightly enhanced by using exogenously applied fluorescent drugs (e.g., 5-aminolevulinic acid induced protoporphyrin IX). Recent advances include the possibility to extract true spectra of single fluorophores (chemical compound that can reemit light upon light excitation) by mathematically eliminating the undesired influences of scattering and absorption. As well, tumour-specific enzymes are about to be specifically targeted by fluorescent markers "smart probes" in order to improve both sensitivity and specificity [54-56]. Ebenezar stated that the diagnostic algorithm based on discriminant function scores obtained by fluorescence excitation spectroscopy (FES) method was able to distinguish welldifferentiated squamous cell carcinoma from normal lesions with a sensitivity of $100 \%$ and specificity of $100 \%$ [57].

3.12.3. Ratio Imaging. This technique compares a photochemical or end metabolic product which is known to be increased in disease status to another product which is known to be depleted. The 5-aminolevulinic acid enhances protoporphyrin IX which fluoresces red after excitation with blue light. The same excitation results in green fluorescence of molecules such as NAD and FADH, which are depleted in high metabolic rate of malignant tissues [54-56]. Shin stated that the sensitivity of the fluorescence imaging techniques ranged from 60 to $97 \%$ and specificity from 75 to $99 \%$ [58].

3.12.4. Raman Spectroscopy. Is laser-based technique that enables chemical characterization and structure of molecules in the sample. It helps to obtain a vibrational spectroscopic picture of the tissue content, thus provide immediate real time histology [54-56]. The technique is used to observe vibrational, rotational, and other low-frequency modes in a system. The Raman effect occurs when light impinges upon a molecule and interacts with the electron cloud and the bonds of that molecule. For the spontaneous Raman effect, which is a form of light scattering, a photon excites the molecule from the ground state to a virtual energy state. When the molecule relaxes it emits a photon and it returns to a different rotational or vibrational state. The difference in energy between the original state and this new state leads to a shift in the emitted photon's frequency away from the excitation wavelength [5456]. The reported sensitivity of this technique was of $80.5 \%$ and specificity of $86.2 \%$ [59].

3.12.5. Elastic Scattering Spectroscopy. The system uses a wide band of wavelengths from $400 \mathrm{~nm}$ up to $700 \mathrm{~nm}$ and recovers the scatter power, scatter amplitude, and absorption species from the reflectance from a 100 micron spot, allowing imaging of tissue at high frame rate. Thus the ESS is the optical signature of the tumour which greatly depends on the 
morphology of the tumour. The probe of the system should be in contact with the tissues, and no light is collected from the surface reflections. Absorption characteristic provides the optical signature of the tissue [54-56]. Lovat et al stated that the sensitivity was $92 \%$ and specificity was $60 \%$ and it differentiated high risk sites from inflammation with a sensitivity and specificity of $79 \%$ [60].

3.12.6. Differential Path-Length Spectroscopy (DPS). Differential path-length spectroscopy is a recently developed fibreoptic point measurement technique that measures scattered photons that have travelled, and their predetermined path lengths are measured. The spectrum is analyzed mathematically and is translated into a set of parameters that are related to the microvasculature and to the intracellular morphology. The reported sensitivity was $69 \%$ and specificity was $85 \%$ [61].

3.12.7. Spectral Scatter Scanning System. The system uses dark field illumination and spectrometer detection in the emission channel together with a scanning mirror. This system has been evaluated for imaging the spectral signature remitted from tissue, with real-time classification algorithm; the system uses a wide band of wavelengths from $400 \mathrm{~nm}$ and up and recovers the scatter power, scatter amplitude, and absorption species from the reflectance from a 100-micron spot; this allows imaging of tissue at high frame rate [62].

3.12.8. Nuclear Magnetic Resonance Spectroscopy. This technology allows three-dimensional study of atoms in the molecule; the larger the magnet, the more sensitive the device. It is possible to view the way of protein link up to DNA [54-56].

3.12.9. Optical Coherence Tomography. It is analogous to ultrasound imaging except that it uses light rather than sound. The high spatial resolution of OCT enables noninvasive in vivo "optical biopsy" and provides immediate and localized diagnostic information. The recent development of a Fourier-domain-mode-lock (FDML) swept source based OCT system helps in achieving a high speed $(>100 \mathrm{kHz}$ Ascan rate) and high spatial resolution $(<4 \mu \mathrm{m})$ simultaneously. In addition, the development of various miniature scanning probes that allow high-speed 3-dimensional OCT picture can be reported [54-56].

3.12.10. Infrared Spectroscopy. It distinguishes different biomolecules by probing chemical bond vibrations and using these molecular and submolecular patterns to define and differentiate pathological from normal tissues [63].

3.12.11. Confocal Endomicroscopy. It is a noninvasive optical biopsy that helps in diagnosis of the pathology of gastrointestinal, dermatologic, and ocular diseases.

3.13. Biomarkers: DNA-Analysis. It is a DNA ploidy analysis after staining with Feulgen dye to determine the malignant potential of cells by comparing with a reference group of cells.
A computer-assisted analysis has been recently designed to identify deviations of cellular DNA content $[48,64]$.

3.14. Laser Capture Microdissection. It is a precise study of cancer biology which helps in defining the molecular basis of malignancy [50]. LCM technology based on extraction of cells from specimens in which the exact morphology of both the captured cells and the surrounding tissue is preserved. When rapid immunohistochemical staining techniques are combined with LCM, more accurate microdissection of cellular subsets can be obtained [65]. It may also help to detect the biomarkers and protein fingerprint models for early detection of oral SCC.

3.15. Lab-on-a-Chip. It is the adaptation, miniaturization, integration, and automation of analytical laboratory procedures into a single device or electronic chip. It utilizes membrane-associated cell proteins to detect dysplastic changes that are singularly expressed on the cell membranes of dysplastic and cancer cells as well as their unique gene transcription profiles [66].

3.16. Imaging Techniques in Diagnosis of Oral SCC. Chest radiography may be indicated because the lungs are the most common site for metastases. It demonstrates metastasis to the lungs or hilar lymph nodes, ribs, or vertebrae. Radiography may be including axial CT scanning or, possibly, other imaging techniques may be needed to determine the degree of spread of some tumours, particularly to exclude bone invasion and lymph node involvement.

Other imaging investigations include MRI or CT scanning of the primary sites of the head and neck and of suspected sites of lymph node or distant metastases.

Bone scanning is of little value in screening because findings are positive only where bone involvement is symptomatic, so radionuclide scanning is occasionally useful. Liver radionuclide scanning shows abnormal findings in as many as $6 \%$ of patients with cancer in the head and neck, but two thirds are false-positive findings; therefore, liver scanning normally is not indicated [15].

Scully stated that the routine panendoscopy helps in identifying simultaneous second primary carcinomas in the esophagus, larynx, or lungs in as many as $14 \%$ of patients. Endoscopy is widely recommended although it is not performed in all centers. More than one-third of SPTs are detectable by endoscopy at or within 1 year of diagnosis of the index tumours [15].

Primary cancers of head and neck, such as HNSCC and non-Hodgkin's lymphoma, are usually staged by MRI or CT. Although MRI is still superior to CT for demonstrating the primary cancer and relationship of cancer boundaries to normal anatomical structures, it is widely believed that CT has advantage in demonstrating bone erosion over the MRI. King stated that artifacts from swallowing, breathing, and coughing limit the quality of MRI and become an increasing problem on moving down the neck. Therefore on descending the head and neck the advantages of MRI decrease and those of CT increase, so in general the MRI is preferred in the 
skull base/nasopharynx and CT in the larynx. The MRI and $\mathrm{CT}$ are usually the modalities of choice for imaging cancers of the aerodigestive tract, US is the method of choice for detecting cancers arising in glandular organs such as the thyroid and salivary glands by guided biopsy performed in the same examination. King stated that FDG-PET has a role in the identification of the unknown primary tumour, but at present it has no proven additional value in staging patients with a known primary tumour [67].

3.17. Functional Imaging Techniques. These techniques are being used to assess the complex interrelated processes in the cancer microenvironment, such as hypoxia, angiogenesis, $\mathrm{pH}$, and metabolism. These techniques have the potential to (1) improve prediction of the cancer behaviour and response to treatment; (2) evaluate new drugs such as the antivascular agents; (3) monitor early cancer response during treatment; and (4) identify residual/recurrent cancer [67].

3.18. Positron Emission Tomography (PET). It is a nuclear medicine imaging technique which produces a threedimensional image or map of functional processes in the body. The system detects pairs of gamma rays emitted indirectly by a positron-emitting radionuclide (tracer), which is introduced into the body on a biologically active molecule. Images of tracer concentration in 3-dimensional space within the body are then reconstructed by computer analysis. Positron emission tomography (PET) is a functional image modality that characterizes the different tissues of the body according to perfusion and metabolic activity of the glucose analogue fludeoxyglucose F 18 ( $\left.{ }^{18} \mathrm{FDG}\right)$. The 18 F-fluoro-2deoxy-D-glucose (FDG) is a radioactively labeled glucose analogue that is utilized due to its capacity to emit positrons, and it can be accurately localized by PET imaging. As tumour cells have an increased uptake of glucose, FDG accumulates within these cells, producing a "hot spot" on the PET image so by this way, it can be distinguished from surrounding normal tissue $[68,69]$. In the head and neck regions, PET showed effectiveness in the detection of unknown primary tumours [70-72]. Positron emission tomography is able to image and identify the location of previously unknown tumours better than any other method, as has been shown in many studies $[70,73,74]$. The most important source of falsepositive results in PET is the inflammatory tissue reaction. Positron emission tomography proved to be the most reliable procedure for detecting recurrent carcinoma.

The utility of PET imaging has been demonstrated in the diagnosis and initial staging of head and neck tumours as well as in the evaluation of persistent or recurrent disease following radiotherapy $[75,76]$. Others have shown the benefit of PET in the detection of unknown primary head and neck cancers or synchronous primary tumours $[77,78]$. One advantage of PET over other imaging modalities, such as computed tomography (CT) or magnetic resonance imaging (MRI), is that since PET imaging visualizes metabolic processes in vivo, relatively small tumours can be detected before structural changes have taken place, as long as they are metabolically active [79]. In fact, previously unapparent tumours, as small as $3 \mathrm{~mm}$, have been detected by PET imaging [80].

PET scans are increasingly read alongside CT or magnetic resonance imaging (MRI) scans; the combination ("coregistration") gives both anatomic and metabolic information (i.e., what the structure is and what it is doing biochemically). Because PET imaging is most useful in combination with anatomical imaging, such as CT, modern PET scanners are now available with integrated high-end multi-detector-row CT scanners. Because the two scans can be performed in immediate sequence during the same session, with the patient not changing position between the two types of scans, the two sets of images are more precisely registered, so that areas of abnormality on the PET imaging can be more perfectly correlated with anatomy on the CT images.

3.19. Diffusion-Weighted Imaging (DWI). DWI is an easy, quick, and widely available functional MR technique. It evaluates the mobility of water in different tissues to generate diffusion weighted images and apparent diffusion coefficient (ADC) maps. Diffusion weighted images are being used to identify a range of cancers, and the $\mathrm{ADC}$ value improves characterization of head and neck tumours, and the ADC of the mass lesions studied had an inverse relationship with lesion cellularity [81-83]. The ADC values can also be used to monitor cancer treatment. During treatment the ADC value of cancer increases as a result of an increase in cell permeability and swelling, cell destruction, leakage of water into extracellular space (EES), and necrosis. These changes may occur early in treatment, preceding volume changes [67].

3.20. Dynamic Contrast-Enhanced Magnetic Resonance Imaging (DCE-MRI). DCE-MRI is performed after the administration of intravenous contrast medium to noninvasively access tumour vascular characteristics. DCE-MRI techniques utilizing low-molecular-weight contrast media have successfully made the transition from methodological development to preclinical and clinical validation and are now rapidly becoming mainstream clinical tools. The low-molecularweight agents $(1000 \mathrm{Da})$ rapidly diffuse in the extracellular fluid (ECF) space [84]. A malignant tumour of a few $\mathrm{mm}^{3}$ in volume cannot rely on the passive diffusion of metabolites from host tissue blood vessels to be able to continue to proliferate, so new vasculature must develop in order for the tumour to continue to grow $[85,86]$. This process of new vascularization or angiogenesis [86] is a signature of neoplasms and one of the principal potential targets for quantitative imaging [87]. It is widely believed that all solid tumours are dependent upon angiogenesis for survival [88], and many antiangiogenic drugs are currently in clinical trials [89]. Thus, methods for imaging and quantitatively assessing this phenomenon will be useful in clinical oncology [90]. The tumour vessels produced by angiogenesis are characteristically leaky, fragile, and incompletely formed unlike mature blood vessels that are the result of normal physiologic processes $[85,86]$. Dynamic contrast-enhanced magnetic resonance imaging (DCE-MRI) is an imaging technique that can measure the density, integrity, and leakiness of tissue vasculature. Changes 
in the parameters obtained by DCE-MRI can be used to assess longitudinal changes within a tumour and, in particular, how a tumour is responding to treatment. The method is based on measurements and mathematical models of how a tracer perfuses through such vessels. Healthy vessels in normal tissues may be characterized by a range of parameters: measuring blood flow, vessel permeability, and tissue volume fractions (i.e., fractions of a given sample of tissue that can be attributed to intravascular or extravascular space). It is hypothesized that some or all of these parameters will be different in pathologic vessels. In recent years, there have been great interest and considerable development in the use of DCEMRI to study these phenomena and test this hypothesis [90].

3.21. Magnetic Resonance (MR) Spectroscopy. It is a noninvasive diagnostic test for measuring biochemical changes in the tumours. While magnetic resonance imaging (MRI) identifies the anatomical location of a tumour, MR spectroscopy compares the chemical composition of normal tissue with abnormal tumour tissue. MR spectroscopy is conducted on the same machine as conventional MRI (see magnetic resonance imaging). The MRI scan uses a powerful magnet, radio waves, and a computer to create detailed images. Spectroscopy is a series of tests that are added to the MRI scan to measure the chemical metabolism of a suspected tumour. MR spectroscopy analyzes molecules such as hydrogen ions or protons. Proton spectroscopy is more commonly used $[91,92]$. There are several different metabolites, or products of metabolism, that can be measured to differentiate between tumour types. MRS provides biochemical information of compounds present in human tissue and cells. Human brain contains hundreds of metabolites, but the proton MRS can only detect a few of them as the least millimolar concentrations are necessary for the metabolites to be detected. The major brain metabolites detected are choline (Cho), creatine (Cr), N-acetyl aspartate (NAA), lactate, myoinositol, glutamine and glutamate, lipids, and the amino acids leucine and alanine [91]. The frequency of these metabolites is measured in units called parts per million (ppm) and plotted on a graph as peaks of varying heights. By measuring each metabolite's ppm and comparing it to normal tissue, the type of tissue present can be determined [92]. MRS demonstrates chemicals or metabolites within cancers that can be used as biomarkers to identify cancer and explore changes associated with hypoxia and cancer treatment. Proton $(\mathrm{H})$ and phosphorus $(\mathrm{P})$ changes in Cho are currently under interrogation for monitoring cancer treatment and have been shown already to be of value in the breast. Lactate is another biomarker that has been found in metastatic nodes from HNSCC and has the potential to be used in the assessment of cancer hypoxia [93].

3.22. Blood Oxygen Level-Dependent MRI (BOLD). It is well known that hypoxic cancers are more resistant to radiotherapy and chemotherapy. Currently there is an interest in improving the therapeutic response of hypoxic cancers to radiotherapy by using radiosensitisers such as carbogen. BOLD is a noninvasive test using MRI that can identify hypoxic cancers (deoxyhaemoglobin) which are likely to respond to this kind of treatment. It uses a T-sensitive sequence during oxygen inhalation to detect an increase in signal resulting from the reduced paramagnetic effect of a reduction in the blood deoxyhaemoglobin within a cancer. As with all functional MR techniques, BOLD presents challenges: the signal is not purely the result of oxygenation, the effects are short lived, and signal changes are small and may be difficult to reproduce. However, despite these difficulties, BOLD has been used successfully to detect decreased hypoxia in the blood (deoxyhaemoglobin in human cancers) during carbogen inhalation $[67,94,95]$ and shows promise for tailoring treatment for hypoxic cancers in the future [67].

\section{Conclusion}

There is wide belief that the OSCC at the anterior site of oral cavity is usually associated with better prognosis than the posterior site; the reason behind this is the sites of lymphatic drainage. The lymphatic involvement considered as the most important determent in prognosis of OSCC. The tumour size is an important factor in lymphatic involvement and it usually affects the choice and outcome of treatment. Tumour thickness is more critical than the size of the tumour in terms of prognosis, and it is closely related to lymph node metastasis. It is believed that it reflects the aggressiveness of tumour growth. The tumour primary site, tumour thickness, and degree of differentiation are the most important determents for lymphatic involvement. The perineural spread is affecting overall prognosis and survival because the perineural and endoneural metastases mean spreading of the tumour cells up to the base of the skull as the tumour cells spread easily though the nerve and the surrounding tissues and compartment. The rapidity of growth, locoregional spread, and distance spread are the factors that reflect the pathological aggressiveness of disease. Distant metastasis was reported in 5-25\% of OSCC patients [29], most commonly in poor locoregional control. Early detection of oral cancer is one of the most efficient ways to reduce the high mortality from this disease. Early detection can minimize the morbidity of the disease and its treatment, which is associated with a severe loss of function, disfigurement, depression, and poor quality of life. There is an urgent need to devise critical diagnostic tools for early detection of oral dysplasia and malignancy that are practical, noninvasive and can be easily performed in an outpatient set-up. Toluidine blue is highly sensitive and moderately specific for malignant lesions and can be used for detection of the suspected areas. The brush biopsy is a reliable technique which equal to surgical biopsy as it collects cells from the full thickness of the oral epithelium. Tissue polypeptide-specific antigen (TPS) and cancer antigen 125 (CA125) are the most critically elevated tumour markers in saliva-based oral cancer test; other tumour markers are insignificantly elevated. Optical diagnostic technologies are techniques that help in early detection of oral dysplasia and malignancy. They are practical, noninvasive and can be easily performed in an outpatient set-up. The chemiluminescence (ViziLite Plus; Microlux/DL, Orascoptic-DK) and Tissue fluorescence imaging (VELscope) are technologies 
based on the fact that the malignant tissues have abnormal metabolic process and different structural changes with different absorbance and reflectance properties for lights than normal tissues. The manufacturers of these screening aids do not make any claim that the device is either sensitive or specific to the identification of any type of abnormal oral lesion. Tissue fluorescence spectroscopy is the most reliable optical diagnostic biopsy with high sensitivity and specificity. It is based on the fact of depletion of NADH and FAD which produce green fluorescence. Enhanced dye fluorescence is based on enhancing protoporphyrin IX which is predominant in the tumour tissues. Raman spectroscopy and elastic scattering spectroscopy are a reliable optical biopsy that provide the optical signature of the tissue by chemical characterization. Optical coherence tomography is analogous to ultrasound imaging except that it uses light rather than sound. DNA-analysis can be used to determine the malignant potential of cells by comparing with a reference group of cells. PET imaging is most useful functional imaging technique in diagnosis of OSCC. FDG-PET has a role in the identification of the unknown primary tumour, but at present it has no proven additional value in staging patients with a known primary tumour. The use of FDG-PET scanning in diagnosis of lymph node involvement is controversial, and there is a wide agreement on using of ultrasound guided fine needle aspiration biopsies in the evaluation of lymph node metastasis, and it is considered as the most reliable technique.

\section{References}

[1] S. L. Parker, T. Tong, S. Bolden, and P. A. Wingo, "Cancer statistics, 1996," Ca-A Cancer Journal for Clinicians, vol. 46, no. 1, pp. 5-27, 1996.

[2] G. E. Laramore, C. B. Scott, M. Al-Sarraf et al., "Adjuvant chemotherapy for resectable squamous cell carcinomas of the head and neck: report on intergroup study 0034," International Journal of Radiation Oncology Biology Physics, vol. 23, no. 4, pp. 705-713, 1992.

[3] S. Petti, M. Masood, G. A. Messano, and C. Scully, "Alcohol is not a risk factor for oral cancer in nonsmoking, betel quid nonchewing individuals. A meta-analysis update," Annali Di Igiene, vol. 25, no. 1, pp. 3-14, 2013.

[4] C.-C. Su and H.-C. Ho, "Neck mass with epithelial papillae: a diagnostic pitfall in aspiration cytology," Otolaryngology-Head and Neck Surgery, vol. 137, no. 6, pp. 979-980, 2007.

[5] B. Rodu and C. Jansson, "Smokeless tobacco and oral cancer: a review of the risks and determinants," Critical Reviews in Oral Biology and Medicine, vol. 15, no. 5, pp. 252-263, 2004.

[6] S. Warnakulasuriya, "Smokeless tobacco and oral cancer," Oral Diseases, vol. 10, no. 1, pp. 1-4, 2004.

[7] C. Scully, "Oral squamous cell carcinoma; from an hypothesis about a virus, to concern about possible sexual transmission," Oral Oncology, vol. 38, no. 3, pp. 227-234, 2002.

[8] S. R. Mallery, G. D. Stoner, P. E. Larsen et al., "Formulation and in-vitro and in-vivo evaluation of a mucoadhesive gel containing freeze dried black raspberries: implications for oral cancer chemoprevention," Pharmaceutical Research, vol. 24, no. 4, pp. 728-737, 2007.

[9] P. Boyle, "Cancer, cigarette smoking and premature death in Europe: a review including the Recommendations of European
Cancer Experts Consensus Meeting, Helsinki, October 1996," Lung Cancer, vol. 17, no. 1, pp. 1-60, 1997.

[10] C. la Vecchia, R. Pagano, and A. Decarli, "Smoking prevalence in younger Italians," Tobacco Control, vol. 5, no. 3, pp. 231-232, 1996.

[11] D. V. Kleinman, P. A. Swango, J. J. Pindborg, and P. Gupta, "Toward assessing trends in oral mucosal lesions: lessons learned from oral cancer," Advances in Dental Research, vol. 7, no. 1, pp. 32-41, 1993.

[12] D. V. Kleinman, P. A. Swango, and J. J. Pindborg, "Epidemiology of oral mucosal lesions in United States schoolchildren: 198687," Community Dentistry and Oral Epidemiology, vol. 22, no. 4, pp. 243-253, 1994.

[13] S. G. Patel and J. P. Shah, "TNM staging of cancers of the head and neck: striving for uniformity among diversity," Ca-A Cancer Journal for Clinicians, vol. 55, no. 4, pp. 242-258, 2005.

[14] C. Scully and R. Bedi, "Ethnicity and oral cancer," The Lancet Oncology, vol. 1, no. 1, pp. 37-42, 2000.

[15] C. Scully and J. Bagan, "Oral squamous cell carcinoma overview," Oral Oncology, vol. 45, no. 4-5, pp. 301-308, 2009.

[16] J. A. Woolgar, "Histopathological prognosticators in oral and oropharyngeal squamous cell carcinoma," Oral Oncology, vol. 42, no. 3, pp. 229-239, 2006.

[17] M. Noguchi, H. Kinjyo, G.-I. Kohama, and K. Nakamori, "Invasive front in oral squamous cell carcinoma: image and flow cytometric analysis with clinicopathologic correlation," Oral Surgery, Oral Medicine, Oral Pathology, Oral Radiology, and Endodontics, vol. 93, no. 6, pp. 682-687, 2002.

[18] P. Garzino-Demo, A. Dell'Acqua, P. Dalmasso et al., "Clinicopathological parameters and outcome of 245 patients operated for oral squamous cell carcinoma," Journal of CranioMaxillofacial Surgery, vol. 34, no. 6, pp. 344-350, 2006.

[19] M. M. Urist, C. J. O’Brien, S.-J. Soong, D. W. Visscher, and W. A. Maddox, "Squamous cell carcinoma of the buccal mucosa: analysis of prognostic factors," American Journal of Surgery, vol. 154, no. 4, pp. 411-414, 1987.

[20] A. R. Shaha, R. H. Spiro, J. P. Shah, and E. W. Strong, "Squamous carcinoma of the floor of the mouth," American Journal of Surgery, vol. 148, no. 4, pp. 455-459, 1984.

[21] H. Platz, R. Fries, M. Hudec, A. M. Tjoa, and R. R. Wagner, "The prognostic relevance of various factors at the time of the first admission of the patient. Retrospective DOSAK study on carcinoma of the oral cavity," Journal of Maxillofacial Surgery, vol. 11, no. 1, pp. 3-12, 1983.

[22] J. D. Crissman, W. Y. Liu, J. L. Gluckman, and G. Cummings, "Prognostic value of histopathologic parameters in squamous cell carcinoma of the oropharynx," Cancer, vol. 54, no. 12, pp. 2995-3001, 1984.

[23] C. Moore, J. G. Kuhns, and R. A. Greenberg, "Thickness as prognostic aid in upper aerodigestive tract cancer," Archives of Surgery, vol. 121, no. 12, pp. 1410-1414, 1986.

[24] C. M. Bier-Laning, R. Durazo-Arvizu, K. Muzaffar, and G. J. Petruzzelli, "Primary tumor thickness as a risk factor for Contralateral cervical metastases in T1/T2 oral tongue squamous cell carcinoma," Laryngoscope, vol. 119, no. 5, pp. 883-888, 2009.

[25] H. Fukano, H. Matsuura, Y. Hasegawa, and S. Nakamura, "Depth of invasion as a predictive factor for cervical lymph node metastasis in tongue carcinoma," Head \& Neck, vol. 19, no. 3, pp. 205-210, 1997.

[26] C. T. Lwin, R. Hanlon, D. Lowe et al., "Accuracy of MRI in prediction of tumour thickness and nodal stage in oral 
squamous cell carcinoma," Oral Oncology, vol. 48, no. 2, pp. 149154, 2012.

[27] J. F. Teichgraeber and A. A. Clairmont, "The incidence of occult metastases for cancer of the oral tongue and floor of the mouth: treatment rationale," Head \& Neck Surgery, vol. 7, no. 1, pp. 1521, 1984

[28] H. Keski-Säntti, T. Atula, J. Törnwall, P. Koivunen, and A. Mäkitie, "Elective neck treatment versus observation in patients with T1/T2 N0 squamous cell carcinoma of oral tongue," Oral Oncology, vol. 42, no. 1, pp. 96-101, 2006.

[29] K. H. Calhoun, P. Fulmer, R. Weiss, and J. A. Hokanson, "Distant metastases from head and neck squamous cell carcinomas," Laryngoscope, vol. 104, no. 10, pp. 1199-1205, 1994.

[30] D. Kademani, R. B. Bell, S. Bagheri et al., "Prognostic factors in intraoral squamous cell carcinoma: the influence of histologic grade," Journal of Oral and Maxillofacial Surgery, vol. 63, no. 11, pp. 1599-1605, 2005.

[31] I. Haberal, H. Çelik, H. Göçmen, H. Akmansu, M. Yörük, and C. Özeri, "Which is important in the evaluation of metastatic lymph nodes in head and neck cancer: palpation, ultrasonography, or computed tomography?" Otolaryngology-Head and Neck Surgery, vol. 130, no. 2, pp. 197-201, 2004.

[32] G. Esen, "Ultrasound of superficial lymph nodes," European Journal of Radiology, vol. 58, no. 3, pp. 345-359, 2006.

[33] E. W. H. To, W. M. Tsang, J. Cheng et al., "Is neck ultrasound necessary for early stage oral tongue carcinoma with clinically N0 neck?” Dentomaxillofacial Radiology, vol. 32, no. 3, pp. 156159, 2003.

[34] M. Knappe, M. Louw, and R. T. Gregor, "Ultrasonographyguided fine-needle aspiration for the assessment of cervical metastases," Archives of Otolaryngology-Head and Neck Surgery, vol. 126, no. 9, pp. 1091-1096, 2000.

[35] N. Anand, N. Chaudhary, M. K. Mittal, and R. Prasad, "Comparison of the efficacy of clinical examination, ultrasound neck and computed tomography in detection and staging of cervical lymph node metastasis in head and neck cancers," Indian Journal of Otolaryngology and Head and Neck Surgery, vol. 59, no. 1, pp. 19-23, 2007.

[36] P. M. Som, “Lymph nodes of theneck," Radiology, vol. 165, no. 3, pp. 593-600, 1987.

[37] A. Ahuja and M. Ying, "Sonography of neck lymph nodes. Part II: abnormal lymph nodes," Clinical Radiology, vol. 58, no. 5, pp. 359-366, 2003.

[38] A. Ahuja, M. Ying, S. F. Leung, and C. Metreweli, "The sonographic appearance and significance of cervical metastatic nodes following radiotherapy for nasopharyngaeal carcinoma," Clinical Radiology, vol. 51, no. 10, pp. 698-701, 1996.

[39] T. S. Atula, M. J. Varpula, T. J. I. Kurki, P.-J. Klemi, and R. Grénman, "Assessment of cervical lymph node status in head and neck cancer patients: palpation, computed tomography and low field magnetic resonance imaging compared with ultrasound-guided fine-needle aspiration cytology," European Journal of Radiology, vol. 25, no. 2, pp. 152-161, 1997.

[40] R. B. J. de Bondt, P. J. Nelemans, P. A. M. Hofman et al., "Detection of lymph node metastases in head and neck cancer: a meta-analysis comparing US, USgFNAC, CT and MR imaging," European Journal of Radiology, vol. 64, no. 2, pp. 266-272, 2007.

[41] A. Mashberg, "Toluidine blue," Journal of the Canadian Dental Association, vol. 61, no. 11, pp. 922-944, 1995.

[42] A. Gupta, M. Singh, R. Ibrahim, and R. Mehrotra, "Utility of toluidine blue staining and brush biopsy in precancerous and cancerous oral lesions," Acta Cytologica, vol. 51, no. 5, pp. 788794, 2007.

[43] I. C. Martin, C. J. Kerawala, and M. Reed, “The application of toluidine blue as a diagnostic adjunct in the detection of epithelial dysplasia," Oral Surgery, Oral Medicine, Oral Pathology, Oral Radiology, and Endodontics, vol. 85, no. 4, pp. 444-446, 1998.

[44] D. Rosenberg and S. Cretin, "Use of meta-analysis to evaluate tolonium chloride in oral cancer screening," Oral Surgery, Oral Medicine, Oral Pathology, vol. 67, no. 5, pp. 621-627, 1989.

[45] J. J. Sciubba, "Improving detection of precancerous and cancerous oral lesions: computer-assisted analysis of the oral brush biopsy," Journal of the American Dental Association, vol. 130, no. 10, pp. 1445-1457, 1999.

[46] C. Scheifele, A.-M. Schmidt-Westhausen, T. Dietrich, and P. A. Reichart, "The sensitivity and specificity of the OralCDx technique: evaluation of 103 cases," Oral Oncology, vol. 40, no. 8, pp. 824-828, 2004.

[47] C. Scully, J. V. Bagan, C. Hopper, and J. B. Epstein, "Oral cancer: current and future diagnostic techniques," American Journal of Dentistry, vol. 21, no. 4, pp. 199-209, 2008.

[48] J. Handschel, D. Öz, N. Pomjanski et al., "Additional use of DNA-image cytometry improves the assessment of resection margins," Journal of Oral Pathology and Medicine, vol. 36, no. 8, pp. 472-475, 2007.

[49] B. G. Zimmermann and D. T. Wong, "Salivary mRNA targets for cancer diagnostics," Oral Oncology, vol. 44, no. 5, pp. 425-429, 2008.

[50] R. Mehrotra, M. Hullmann, R. Smeets, T. E. Reichert, and O. Driemel, "Oral cytology revisited," Journal of Oral Pathology and Medicine, vol. 38, no. 2, pp. 161-166, 2009.

[51] C. S. Betz, T. Makriniotis, H. Stepp et al., "Diagnosis of head \& neck malignancy using fluorescence spectroscopy and imaging," Head \& Neck Oncology, vol. 1, supplement 1, article O4, 2009.

[52] H. Stepp, A. Johansson, C. S. Betz, and A. Leunig, "Fluorescence spectroscopy and fluorescence imaging for tissue diagnosticsprinciples and methods," Head \& Neck Oncology, vol. 1, supplement 1, article O3, 2009.

[53] B. D. Kulbersh, R. D. Duncan, J. S. Magnuson, J. B. Skipper, K. Zinn, and E. L. Rosenthal, "Sensitivity and specificity of fluorescent immunoguided neoplasm detection in head and neck cancer xenografts," Archives of Otolaryngology-Head and Neck Surgery, vol. 133, no. 5, pp. 511-515, 2007.

[54] M. A. A. Suhr, C. Hopper, L. Jones, J. G. D. George, S. G. Bown, and A. J. MacRobert, "Optical biopsy systems for the diagnosis and monitoring of superficial cancer and precancer," International Journal of Oral and Maxillofacial Surgery, vol. 29, no. 6, pp. 453-457, 2000.

[55] T. Upile, W. Jerjes, H. J. C. M. Sterenborg et al., "Head \& neck optical diagnostics: vision of the future of surgery," Head \& Neck Oncology, vol. 1, article 25, 2009.

[56] W. Jerjes, T. Upile, A. Petrie et al., "Clinicopathological parameters, recurrence, locoregional and distant metastasis in 115 T1-T2 oral squamous cell carcinoma patients," Head \& Neck Oncology, vol. 2, article 9, 2010.

[57] J. Ebenezar, S. Ganesan, P. Aruna, R. Muralinaidu, K. Renganathan, and T. R. Saraswathy, "Noninvasive fluorescence excitation spectroscopy for the diagnosis of oral neoplasia in vivo," Journal of Biomedical Optics, vol. 17, no. 9, Article ID 97007-1, 2012. 
[58] D. Shin, N. Vigneswaran, A. Gillenwater, and R. RichardsKortum, "Advances in fluorescence imaging techniques to detect oral cancer and its precursors," Future Oncology, vol. 6, no. 7, pp. 1143-1154, 2010.

[59] S. Duraipandian, M. S. Bergholt, W. Zheng et al., "Real-time Raman spectroscopy for in vivo, online gastric cancer diagnosis during clinical endoscopic examination," Journal of Biomedical Optics, vol. 17, no. 8, Article ID 081418, 2012.

[60] L. B. Lovat, K. Johnson, G. D. Mackenzie et al., "Elastic scattering spectroscopy accurately detects high grade dysplasia and cancer in Barrett's oesophagus," Gut, vol. 55, no. 8, pp. 10781083, 2006.

[61] H. J. C. M. Sterenborg, M. J. H. Witjes, S. de Visscher, and A. Amelink, "Differential pathlength spectroscopy for diagnosis of head and neck cancer," Head \& Neck Oncology, vol. 1, supplement 1, article O7, 2009.

[62] B. W. Pogue, V. Krishnaswamy, A. Laughney, K. D. Paulsen, P. J. Hoopes, and P. B. G. Allende, "Spectral scatter scanning system for surgical margin detection," Head \& Neck Oncology, vol. 1, supplement 1, article O12, 2009.

[63] C. Conti, P. Ferraris, E. Giorgini, C. Rubini, S. Sabbatini, and G. Tosi :, "Microimaging FT-IR of head and neck tumours. The case of salivary glands," Head \& Neck Oncology, vol. 1, supplement 1, article O13, 2009.

[64] G. Bradley, E. W. Odell, S. Raphael et al., "Abnormal DNA content in oral epithelial dysplasia is associated with increased risk of progression to carcinoma," British Journal of Cancer, vol. 103, no. 9, pp. 1432-1442, 2010.

[65] F. Fend, M. R. Emmert-Buck, R. Chuaqui et al., "ImmunoLCM: laser capture microdissection of immunostained frozen sections for mRNA analysis," American Journal of Pathology, vol. 154, no. 1, pp. 61-66, 1999.

[66] B. L. Ziober, M. G. Mauk, E. M. Falls, Z. Chen, A. F. Ziober, and H. H. Bau, "Lab-on-a-chip for oral cancer screening and diagnosis," Head \& Neck, vol. 30, no. 1, pp. 111-121, 2008.

[67] A. D. King, "Multimodality imaging of head and neck cancer," Cancer Imaging, vol. 7, no. A, pp. S37-S46, 2007.

[68] K. E. Rusthoven, M. Koshy, and A. C. Paulino, "The role of fluorodeoxyglucose positron emission tomography in cervical lymph node metastases from an unknown primary tumor," Cancer, vol. 101, no. 11, pp. 2641-2649, 2004.

[69] E. J. Mahoney and J. H. Spiegel, "Evaluation and management of malignant cervical lymphadenopathy with an unknown primary tumor," Otolaryngologic Clinics of North America, vol. 38, no. 1, pp. 87-97, 2005.

[70] M. M. Hanasono, L. D. Kunda, G. M. Segall, G. H. Ku, and D. J. Terris, "Uses and limitations of FDG positron emission tomography in patients with head and neck cancer," The Laryngoscope, vol. 109, no. 6, pp. 880-885, 1999.

[71] A. C. Kole, O. E. Nieweg, J. Pruim et al., "Detection of unknow occult primary tumors using positron emission tomography," Cancer, vol. 82, no. 6, pp. 1160-1166, 1998.

[72] W. M. Mendenhall, A. A. Mancuso, J. T. Parsons, S. P. Stringer, and N. J. Cassisi, "Diagnostic evaluation of squamous cell carcinoma metastatic to cervical lymph nodes from an unknown head and neck primary site," Head \& Neck, vol. 20, no. 8, pp. 739-744, 1998.

[73] A. C. Kole, O. E. Nieweg, J. Pruim et al., "Detection of unknow occult primary tumors using positron emission tomography," Cancer, vol. 82, no. 6, pp. 1160-1166, 1998.
[74] A. A. Safa, L. M. Tran, S. Rege et al., "The role of positron emission tomography in occult primary head and neck cancers," The Cancer Journal From Scientific American, vol. 5, no. 4, pp. 214-218, 1999.

[75] E. di Martino, B. Nowak, H. A. Hassan et al., "Diagnosis and staging of head and neck cancer: a comparison of modern imaging modalities (positron emission tomography, computed tomography, color-coded duplex sonography) with panendoscopic and histopathologic findings," Archives of Otolaryngology-Head and Neck Surgery, vol. 126, no. 12, pp. 1457-1461, 2000.

[76] K. M. Greven, D. W. Williams III, W. F. McGuirt Sr. et al., "Serial positron emission tomography scans following radiation therapy of patients with head and neck cancer," Head \& Neck, vol. 23, no. 11, pp. 942-946, 2001.

[77] M. Jungehülsing, K. Scheidhauer, M. Damm et al., "2[18F]fluoro-2-deoxy-D-glucose positron emission tomography is a sensitive tool for the detection of occult primary cancer (carcinoma of unknown primary syndrome) with head and neck lymph node manifestation," Otolaryngology-Head and Neck Surgery, vol. 123, no. 3, pp. 294-301, 2000.

[78] M. P. Stokkel, K. G. Moons, F. W. ten Broek, P. P. van Rijk, and G. J. Hordijk, "18 F-Fluorodeoxyglucose dual-head positron emission tomography as a procedure for detecting simultaneous primary tumors in cases of head and neck cancer," Cancer, vol. 86, no. 11, pp. 2370-2377, 1999.

[79] S. C. Rankin, "PET in face and neck tumours," Cancer Imaging, vol. 6, no. A, pp. S89-S95, 2006.

[80] O. S. Aassar, N. J. Fischbein, G. R. Caputo et al., "Metastatic head and neck cancer: role and usefulness of FDG PET in locating occult primary tumors," Radiology, vol. 210, no. 1, pp. 177-181, 1999.

[81] M. Maeda, H. Kato, H. Sakuma, S. E. Maier, and K. Takeda, "Usefulness of the apparent diffusion coefficient in line scan diffusion-weighted imaging for distinguishing between squamous cell carcinomas and malignant lymphomas of the head and neck," American Journal of Neuroradiology, vol. 26, no. 5, pp. 1186-1192, 2005.

[82] M. Sumi, N. Sakihama, T. Sumi et al., "Discrimination of metastatic cervical lymph nodes with diffusion-weighted MR imaging in patients with head and neck cancer," American Journal of Neuroradiology, vol. 24, no. 8, pp. 1627-1634, 2003.

[83] C. R. Habermann, P. Gossrau, J. Graessner et al., "Diffusionweighted echo-planar MRI: a valuable tool for differentiating primary parotid gland tumors?” Rofo, vol. 177, no. 7, pp. 940945, 2005.

[84] A. R. Padhani, "Dynamic contrast-enhanced MRI in clinical oncology: current status and future directions," Journal of Magnetic Resonance Imaging, vol. 16, no. 4, pp. 407-422, 2002.

[85] D. Ribatti, A. Vacca, and M. Presta, "The discovery of angiogenic factors: a historical review," General Pharmacology, vol. 35, no. 5, pp. 227-231, 2000.

[86] J. Folkman, "Angiogenesis in cancer, vascular, rheumatoid and other disease," Nature Medicine, vol. 1, no. 1, pp. 27-31, 1995.

[87] M. Atri, "New technologies and directed agents for applications of cancer imaging," Journal of Clinical Oncology, vol. 24, no. 20, pp. 3299-3308, 2006.

[88] P. Carmeliet and R. K. Jain, "Angiogenesis in cancer and other diseases," Nature, vol. 407, no. 6801, pp. 249-257, 2000.

[89] C. Verhoef, J. H. W. de Wilt, and H. M. W. Verheul, "Angiogenesis inhibitors: perspectives for medical, surgical and radiation 
oncology," Current Pharmaceutical Design, vol. 12, no. 21, pp. 2623-2630, 2006.

[90] T. E. Yankeelov and J. C. Gore, "Dynamic contrast enhanced magnetic resonance imaging in oncology: theory, data acquisition, analysis, and examples," Current Medical Imaging Reviews, vol. 3, no. 2, pp. 91-107, 2009.

[91] C. Majós, C. Aguilera, M. Cos et al., "In vivo proton magnetic resonance spectroscopy of intraventricular tumours of the brain," European Radiology, vol. 19, no. 8, pp. 2049-2059, 2009.

[92] D. A. Sanghvi, "Recent advances in imaging of brain tumors," Indian Journal of Cancer, vol. 46, no. 2, pp. 82-87, 2009.

[93] A. A. K. A. Razek, L. G. Elsorogy, N. Y. Soliman, and N. Nada, "Dynamic susceptibility contrast perfusion MR imaging in distinguishing malignant from benign head and neck tumors: a pilot study," European Journal of Radiology, vol. 77, no. 1, pp. 73-79, 2011.

[94] J. R. Griffiths, N. J. Taylor, F. A. Howe et al., "The response of human tumors to carbogen breathing, monitored by gradientrecalled echo magnetic resonance imaging," International Journal of Radiation Oncology Biology Physics, vol. 39, no. 3, pp. 697701, 1997.

[95] M. Rijpkema, J. H. A. M. Kaanders, F. B. M. Joosten, A. J. van der Kogel, and A. Heerschap, "Effects of breathing a hyperoxic hypercapnic gas mixture on blood oxygenation and vascularity of head-and-neck tumors as measured by magnetic resonance imaging," International Journal of Radiation Oncology Biology Physics, vol. 53, no. 5, pp. 1185-1191, 2002. 


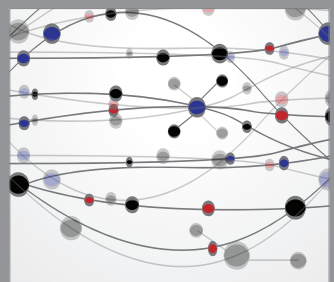

The Scientific World Journal
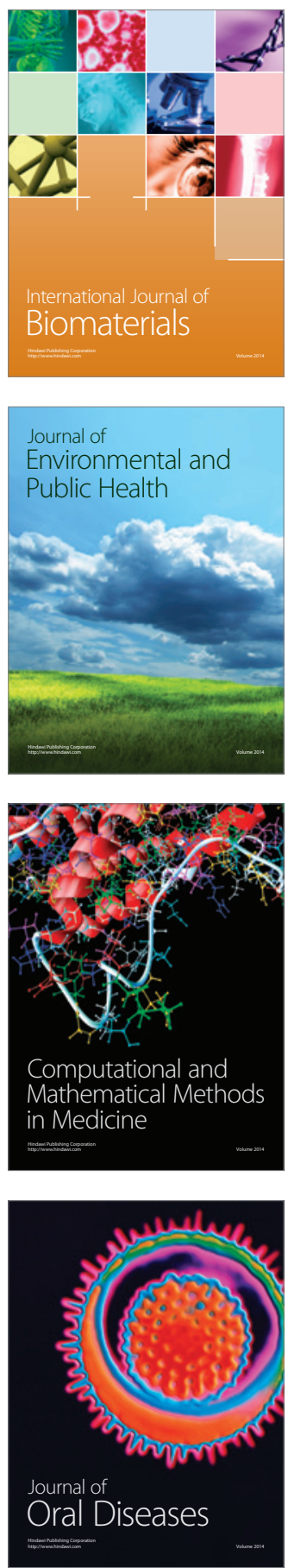
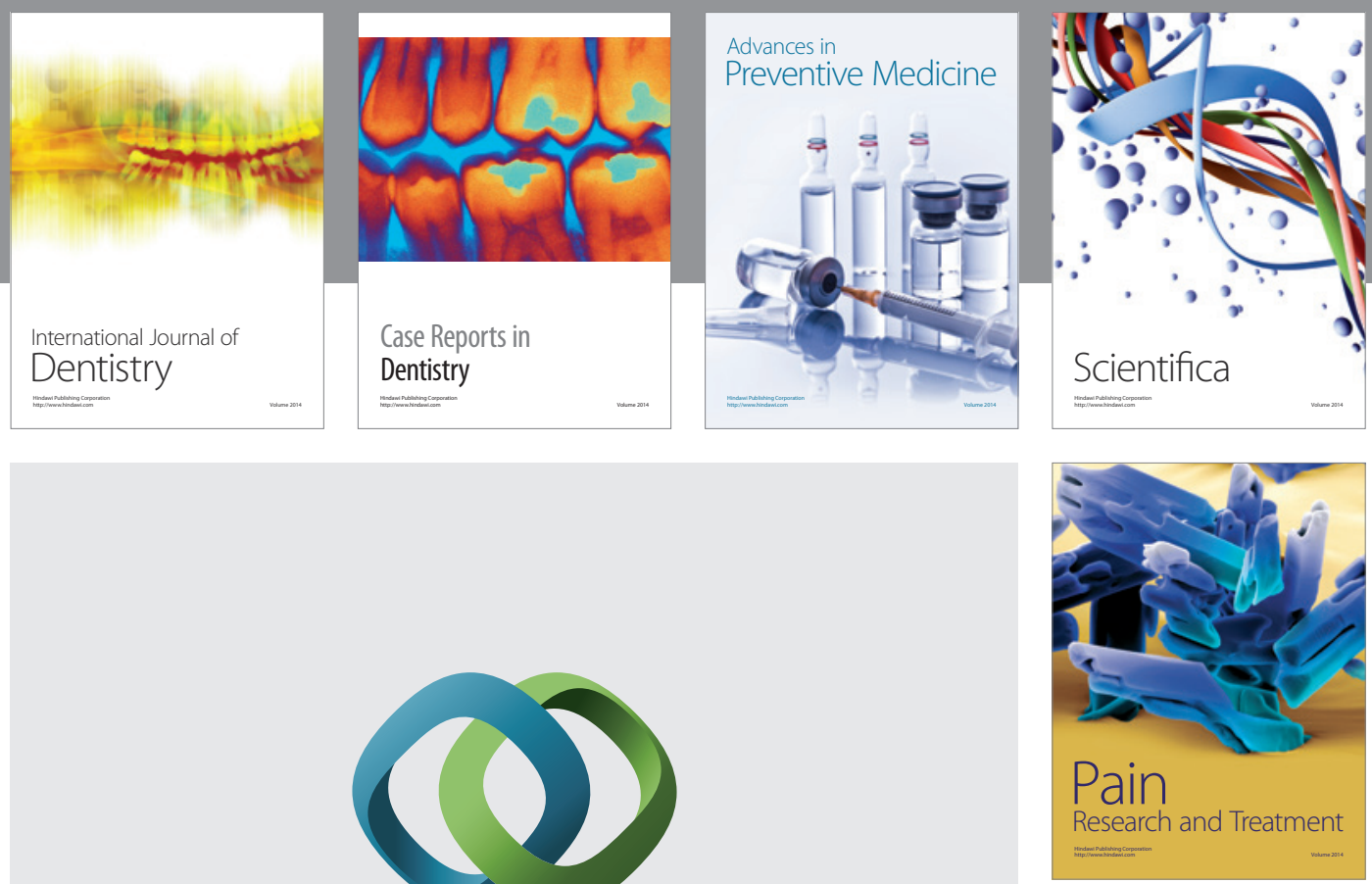

\section{Hindawi}

Submit your manuscripts at

http://www.hindawi.com
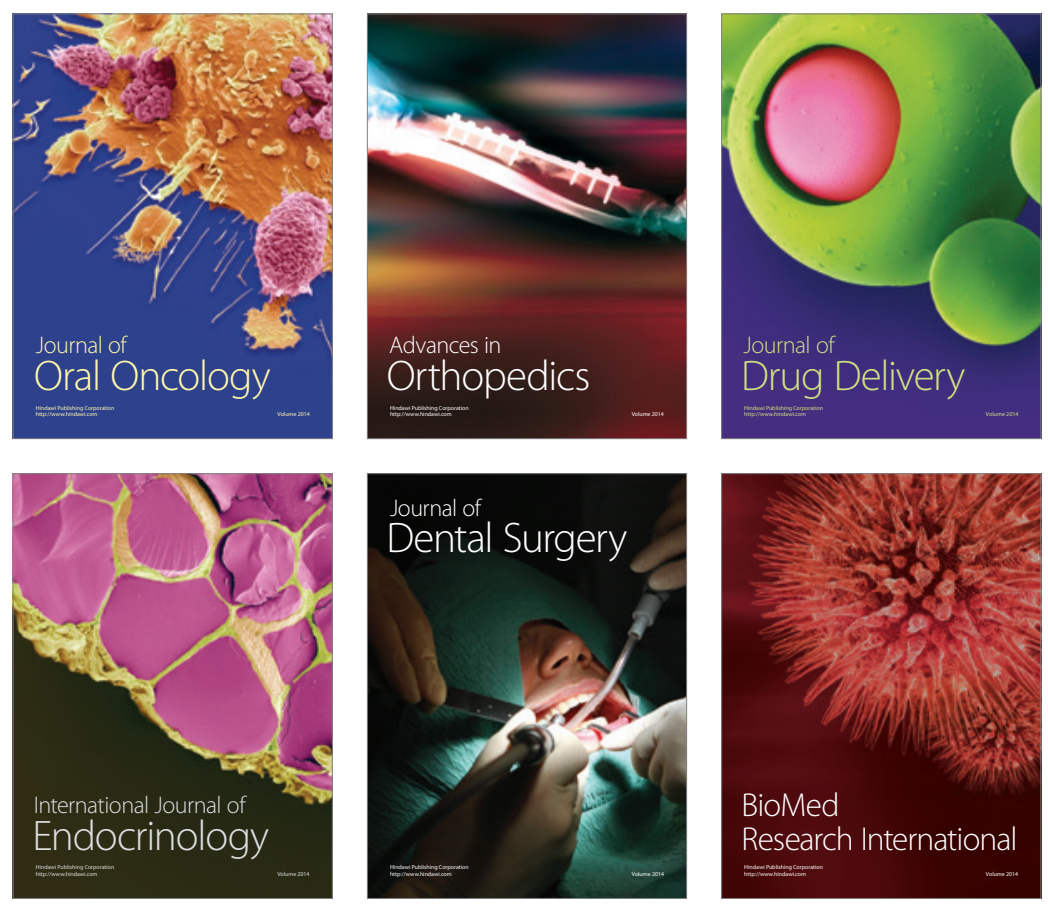

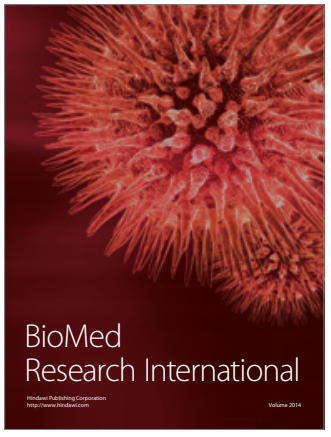

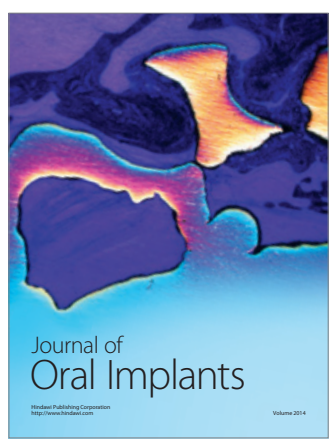
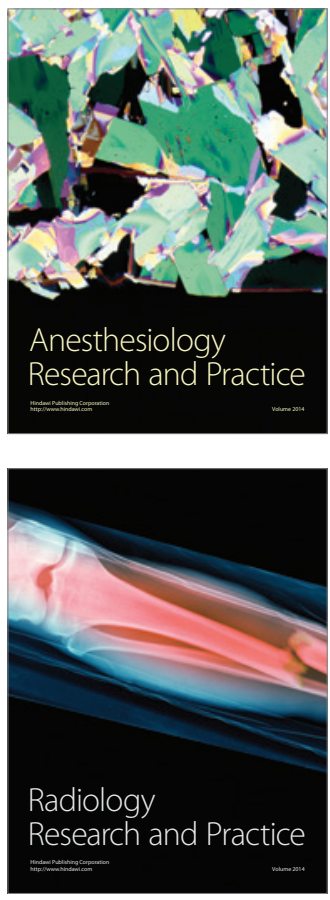